\title{
A GENERALIZATION OF KY FAN'S INEQUALITY
}

\section{PENG GAO}

(Received 20 March 2001 and in revised form 17 July 2001)

\begin{abstract}
Let $P_{n, r}(\mathbf{x})$ be the generalized weighted means. Let $F(x)$ be a $C^{1}$ function, $y=y(x)$ an implicit decreasing function defined by $f(x, y)=0$ and $0<m<M \leq m^{\prime}$, $n \geq 2, x_{i} \in[m, M], y_{i} \in\left[m^{\prime}, M^{\prime}\right]$. Then for $-1 \leq r \leq 1$, if $f_{x}^{\prime} / f_{y}^{\prime} \leq 1, \mid\left(F\left(P_{n, 1}(\mathbf{y})\right)-\right.$ $\left.F\left(P_{n, r}(\mathbf{y})\right)\right) /\left(F\left(P_{n, 1}(\mathbf{x})\right)-F\left(P_{n, r}(\mathbf{x})\right)\right) \mid<\left(\max _{m^{\prime} \leq \xi \leq M^{\prime}}\left|F^{\prime}(\xi)\right|\right) /\left(\min _{m \leq \eta \leq M}\left|F^{\prime}(\eta)\right|\right)$. $M / m^{\prime}$. A similar result exists for $f_{x}^{\prime} / f_{y}^{\prime} \geq 1$. By specifying $f(x, y)$ and $F(x)$, we get various generalizations of Ky Fan's inequality. We also present some results on the comparison of $P_{n, s}^{\alpha}(\mathbf{y})-P_{n, r}^{\alpha}(\mathbf{y})$ and $P_{n, s}^{\alpha}(\mathbf{x})-P_{n, r}^{\alpha}(\mathbf{x})$ for $s \geq r, \alpha \in \mathbb{R}$.
\end{abstract}

2000 Mathematics Subject Classification. 26D15, 26D20.

1. Introduction. Let $P_{n, r}(\mathbf{x})$ be the generalized weighted means defined by $P_{n, r}(\mathbf{x})=$ $\left(\sum_{i=1}^{n} \omega_{i} x_{i}^{r}\right)^{1 / r}$, where $w_{i}, 1 \leq i \leq n$ are positive real numbers with $\sum_{i=1}^{n} w_{i}=1$ and $\mathbf{x}=\left(x_{1}, x_{2}, \ldots, x_{n}\right)$. Here we denote $P_{n, 0}(\mathbf{x})$ as $\lim _{r \rightarrow 0^{+}} P_{n, r}(\mathbf{x})$. Let $f(x, y)$ be a real function, we write $f(\mathbf{x}, \mathbf{y})=0$ for $\mathbf{y}=\left(y_{1}, y_{2}, \ldots, y_{n}\right)$ if for all $i=1, \ldots, n, f\left(x_{i}, y_{i}\right)=0$.

In this paper, we always assume $x_{1} \leq x_{2} \leq \cdots \leq x_{n}, f(\mathbf{x}, \mathbf{y})=0$ and denote $x_{1}=m$, $x_{n}=M, y_{1}=M^{\prime}, y_{n}=m^{\prime}$. We also write $A_{n}=P_{n, 1}(\mathbf{x}), G_{n}=P_{n, 0}(\mathbf{x}), H_{n}=P_{n,-1}(\mathbf{x})$, $A_{n}^{\prime}=P_{n, 1}(\mathbf{y}), G_{n}^{\prime}=P_{n, 0}(\mathbf{y}), H_{n}^{\prime}=P_{n,-1}(\mathbf{y})$.

The following inequality, originally due to Ky Fan, was first published in the monograph Inequalities by Beckenbach and Bellman [6, page 5].

THEOREM 1.1. For $f(x, y)=x+y-1, x_{i} \in[0,1 / 2]$,

$$
\frac{A_{n}^{\prime}}{G_{n}^{\prime}} \leq \frac{A_{n}}{G_{n}}
$$

with equality holding if and only if $x_{1}=\cdots=x_{n}$.

Ky Fan's inequality has evoked the interest of several mathematicians and many papers appeared providing new proofs, generalizations and sharpenings of (1.1). We refer the reader to the survey article [3] and the references therein.

Under the same condition of Theorem 1.1, the following additive analogue of (1.1) was proved by Alzer [1].

THEOREM 1.2. For $f(x, y)=x+y-1, x_{i} \in[0,1 / 2]$,

$$
A_{n}^{\prime}-G_{n}^{\prime} \leq A_{n}-G_{n}
$$

with equality holding if and only if $x_{1}=\cdots=x_{n}$.

Refinements of (1.1) and (1.2), were obtained by Alzer (see [4, 5]) in the following two theorems, respectively. 
THEOREM 1.3. Let $x_{i} \in(0,1 / 2](i=1,2, \ldots, n ; n \geq 2)$ and $m<M$, then

$$
\frac{m}{1-m}<\frac{A_{n}^{\prime}-G_{n}^{\prime}}{A_{n}-G_{n}}<\frac{M}{1-M} .
$$

THEOREM 1.4. Let $x_{i} \in[a, b](i=1,2, \ldots, n ; 0<a<b<1)$, then

$$
\left(\frac{A_{n}}{G_{n}}\right)^{(a /(1-a))^{2}}<\frac{A_{n}^{\prime}}{G_{n}^{\prime}}<\left(\frac{A_{n}}{G_{n}}\right)^{(b /(1-b))^{2}} .
$$

Recently, Mercer obtained the following generalized Ky Fan's inequality [7].

THEOREM 1.5. For $f(x, y)=x^{p}+y^{p}-1, p \geq 1, n \geq 2, x_{i} \in\left[0,2^{-(1 / p)}\right]$,

$$
P_{n, 1}(\mathbf{x}) P_{n, 0}(\mathbf{y}) \geq P_{n, 1}(\mathbf{y}) P_{n, 0}(\mathbf{x})
$$

with equality holding if and only if $x_{1}=\cdots=x_{n}$.

Our main goal in this paper is to present a theorem which provides essentially a unified treatment of Theorems 1.1, 1.2, 1.3, 1.4, and 1.5 and also gives new extensions of Ky Fan's inequality. In Section 3, applications to Ky Fan's inequality will be given by specifying the functions $f(x, y), F(x)$.

More generally, we can talk about the comparison of $P_{n, s}^{\alpha}(\mathbf{y})-P_{n, r}^{\alpha}(\mathbf{y})$ and $P_{n, s}^{\alpha}(\mathbf{x})-$ $P_{n, r}^{\alpha}(\mathbf{x})$ for real $\alpha$. The case of $A_{n}^{\prime \alpha}-G_{n}^{\prime \alpha}$ and $A_{n}^{\alpha}-G_{n}^{\alpha}$ was discussed in [5] and we will give some results related to the general case in Section 4.

\section{The main theorem}

THEOREM 2.1. Let $F(x)$ be a $C^{1}$ function, $y=y(x)$ an implicit decreasing function defined by $f(x, y)=0$ and $0<m<M \leq m^{\prime}, n \geq 2$. Then for $-1 \leq r \leq 1$, if $f_{x} / f_{y} \leq 1$,

$$
\left|\frac{F\left(P_{n, 1}(\mathbf{y})\right)-F\left(P_{n, r}(\mathbf{y})\right)}{F\left(P_{n, 1}(\mathbf{x})\right)-F\left(P_{n, r}(\mathbf{x})\right)}\right|<\frac{\max _{m^{\prime} \leq \xi \leq M^{\prime}}\left|F^{\prime}(\xi)\right|}{\min _{m \leq \eta \leq M}\left|F^{\prime}(\eta)\right|} \cdot \frac{M}{m^{\prime}} .
$$

If $f_{x}^{\prime} / f_{y}^{\prime} \geq 1$

$$
\frac{\min _{m^{\prime} \leq \xi \leq M^{\prime}}\left|F^{\prime}(\xi)\right|}{\max _{m \leq \eta \leq M}\left|F^{\prime}(\eta)\right|} \cdot \frac{m}{M^{\prime}}<\left|\frac{F\left(P_{n, 1}(\mathbf{y})\right)-F\left(P_{n, r}(\mathbf{y})\right)}{F\left(P_{n, 1}(\mathbf{x})\right)-\left(P_{n, r}(\mathbf{x})\right)}\right|
$$

provided the denominators on both sides are nonzero.

Proof. Since the proofs of (2.1) and (2.2) are very similar, we only prove (2.1) for $r \neq 0$ here, the case $r=0$ is also similar. We will consider the case $F(x)=x$ first. We define for $1 \leq i \leq n-1$ and $0<x<x_{i+1}$ :

$$
\begin{aligned}
\mathbf{x}_{i} & =\left(x, \ldots, x, x_{i+1}, \ldots, x_{n}\right), \\
\mathbf{y}_{i} & =\left(y, \ldots, y, y_{i+1}, \ldots, y_{n}\right), \\
D\left(\mathbf{x}_{i}\right) & =x_{n}\left(P_{n, 1}\left(\mathbf{x}_{i}\right)-P_{n, r}\left(\mathbf{x}_{i}\right)\right)-y_{n}\left(P_{n, 1}\left(\mathbf{y}_{i}\right)-P_{n, r}\left(\mathbf{y}_{i}\right)\right), \\
g\left(\mathbf{x}_{i}\right) & =x_{n} P_{n, r}\left(\mathbf{x}_{i}\right)^{1-r} \cdot x^{r-1}+y_{n} P_{n, r}\left(\mathbf{y}_{i}\right)^{1-r} \cdot y^{r-1}
\end{aligned}
$$

and $D_{i}(x)=D\left(\mathbf{x}_{i}\right), g_{i}(x)=g\left(\mathbf{x}_{i}\right)$. 
We need to show that $D_{1}\left(x_{1}\right)>0$. Differentiation of $D_{1}\left(x_{1}\right)$ yields

$$
\begin{aligned}
\Omega_{i}^{-1} D_{i}^{\prime}(x) & =x_{n}\left(1-P_{n, r}\left(\mathbf{x}_{i}\right)^{1-r} \cdot x^{r-1}\right)+y_{n} \frac{f_{x}^{\prime}}{f_{y}^{\prime}}\left(1-P_{n, r}\left(\mathbf{y}_{i}\right)^{1-r} \cdot y^{r-1}\right) \\
& \leq x_{n}\left(1-P_{n, r}\left(\mathbf{x}_{i}\right)^{1-r} \cdot x^{r-1}\right)+y_{n}\left(1-P_{n, r}\left(\mathbf{y}_{i}\right)^{1-r} \cdot y^{r-1}\right) \\
& =x_{n}+y_{n}-g_{i}(x),
\end{aligned}
$$

where $\Omega_{i}=\sum_{j=1}^{i} \omega_{i}$.

Consider

$$
\begin{aligned}
g_{i}^{\prime}(x) & =-(1-r) \sum_{j=i+1}^{n} \omega_{j}\left[\left(\frac{P_{n, r}\left(\mathbf{x}_{i}\right)}{x}\right)^{1-2 r} \cdot \frac{x_{n} x_{j}^{r}}{x^{r+1}}-\frac{f_{x}^{\prime}}{f_{y}^{\prime}} \cdot\left(\frac{P_{n, r}\left(\mathbf{y}_{i}\right)}{y}\right)^{1-2 r} \cdot \frac{y_{n} y_{j}^{r}}{y^{r+1}}\right] \\
& \leq-(1-r) \sum_{j=i+1}^{N} \omega_{j}\left[\left(\frac{P_{n, r}\left(\mathbf{x}_{i}\right)}{x}\right)^{1-2 r} \cdot \frac{x_{n} x_{j}^{r}}{x^{r+1}}-\left(\frac{P_{n, r}\left(\mathbf{y}_{i}\right)}{y}\right)^{1-2 r} \cdot \frac{y_{n} y_{j}^{r}}{y^{r+1}}\right]<0 .
\end{aligned}
$$

The last inequality holds, since when $-1 \leq r \leq 1 / 2, k=i+1, \ldots, n$, we have

$$
\begin{gathered}
\left(\frac{P_{n, r}\left(\mathbf{x}_{i}\right)}{x}\right)^{1-2 r} \geq\left(\frac{P_{n, r}\left(\mathbf{y}_{i}\right)}{y}\right)^{1-2 r}, \quad \frac{x_{k}}{x}>\frac{y_{k}}{y}, \\
\frac{x_{n}}{y_{n}} \cdot\left(\frac{x_{j}}{y_{j}}\right)^{r} \geq\left(\frac{x_{j}}{y_{j}}\right)^{1+r}>\left(\frac{x}{y}\right)^{1+r},
\end{gathered}
$$

when $1 / 2 \leq r \leq 1$, we have

$$
\begin{gathered}
\left(\frac{P_{n, r}\left(\mathbf{x}_{i}\right)}{x}\right)^{1-2 r} \geq\left(\frac{x_{n}}{x}\right)^{1-2 r}, \quad\left(\frac{P_{n, r}\left(\mathbf{y}_{i}\right)}{y}\right)^{1-2 r} \leq\left(\frac{y_{n}}{y}\right)^{1-2 r}, \\
\left(\frac{x_{n}}{y_{n}}\right)^{2-2 r} \cdot\left(\frac{x_{j}}{y_{j}}\right)^{r} \geq\left(\frac{x_{j}}{y_{j}}\right)^{2-2 r} \cdot\left(\frac{x_{j}}{y_{j}}\right)^{r}=\left(\frac{x_{j}}{y_{j}}\right)^{2-r}>\left(\frac{x}{y}\right)^{2-r} .
\end{gathered}
$$

Thus $g_{i}(x)>g_{i}\left(x_{i+1}\right)=g_{i+1}\left(x_{i+1}\right) \geq g_{i+1}\left(x_{i+2}\right) \geq \cdots \geq g_{n-1}\left(x_{n-1}\right) \geq g_{n-1}\left(x_{n}\right)=$ $x_{n}+y_{n}$, which implies $D_{i}^{\prime}(x)<0$ for all $x \in\left(0, x_{i+1}\right)$, so

$$
D_{1}\left(x_{1}\right) \geq D_{1}\left(x_{2}\right)=D_{2}\left(x_{2}\right) \geq D_{2}\left(x_{3}\right) \geq \cdots \geq D_{n-1}\left(x_{n-1}\right) \geq D_{n-1}\left(x_{n}\right)=0 .
$$

Since $D_{i}$ is strictly decreasing, we conclude from $m<M$ that $D_{1}\left(x_{1}\right)>0$.

Next, for arbitrary $F$, by using the mean value theorem, (2.1) is equivalent to

$$
\frac{F\left(P_{n, 1}(\mathbf{y})\right)-F\left(P_{n, r}(\mathbf{y})\right)}{F\left(P_{n, 1}(\mathbf{x})\right)-F\left(P_{n, r}(\mathbf{x})\right)}=\frac{F^{\prime}(\xi)}{F^{\prime}(\eta)} \cdot \frac{P_{n, 1}(\mathbf{y})-P_{n, r}(\mathbf{y})}{P_{n, 1}(\mathbf{x})-P_{n, r}(\mathbf{x})},
$$

where $m^{\prime} \leq \xi \leq M^{\prime}, m \leq \eta \leq M$. Taking absolute value and applying the result for $F(x)=x$, we get the desired inequality (2.1). This completes the proof. 
3. Consequences of Theorem 2.1. In this section, by choosing different functions $f(x, y), F(x)$, we will give several results of generalized Ky Fan's inequality of type (2.1). There are corresponding ones of type (2.2) and we leave the statements to the reader. To simplify expressions, we define

$$
\Delta_{s, r, \alpha}=\frac{P_{n, s}^{\alpha}(\mathbf{y})-P_{n, r}^{\alpha}(\mathbf{y})}{P_{n, s}^{\alpha}(\mathbf{x})-P_{n, r}^{\alpha}(\mathbf{x})}
$$

with

$$
\Delta_{s, r, 0}=\frac{\left(\ln \left(P_{n, s}(\mathbf{y}) / P_{n, r}(\mathbf{y})\right)\right)}{\left(\ln \left(P_{n, s}(\mathbf{x}) / P_{n, r}(\mathbf{x})\right)\right)} .
$$

Also in order to include the case of equality for various inequalities in our discussion, we define $0 / 0=1$ from now on.

As a generalization of Theorem 1.3, we have the following corollary.

COROLlarY 3.1. Let $f(x, y)=a x^{p}+b y^{p}-1,0<a \leq b, p \geq 1,0<m<M \leq$ $(a+b)^{-(1 / p)}, n \geq 2$. For $\alpha \leq 1$, let $F(x)=\ln x$ if $\alpha=0$, or $F(x)=x^{\alpha}$, otherwise. Then for $-1 \leq r<1$

$$
\Delta_{1, r, \alpha}<\left(\frac{M}{m^{\prime}}\right)^{2-\alpha} .
$$

Proof. This follows from $f_{x}^{\prime} / f_{y}^{\prime} \leq 1, \max _{m^{\prime} \leq \xi \leq M^{\prime}}\left|F^{\prime}(\xi)\right| / \min _{m \leq \eta \leq M}\left|F^{\prime}(\eta)\right| \leq$ $\left(M / m^{\prime}\right)^{1-\alpha}$.

We remark here in Corollary 3.1, the case $\alpha=0$ gives $P_{n, 1}(\mathbf{y}) / P_{n, r}(\mathbf{y})<\left(P_{n, 1}(\mathbf{x}) /\right.$ $\left.P_{n, r}(\mathbf{x})\right)^{\left(M / m^{\prime}\right)^{2}}$, which partially generalizes Theorem 1.4. Also for the case $\alpha=0$, by only assuming $x_{i} \in\left[0,(a+b)^{-(1 / p)}\right]$, we get $P_{n, 1}(\mathbf{y}) / P_{n, r}(\mathbf{y}) \leq P_{n, 1}(\mathbf{x}) / P_{n, r}(\mathbf{x})$ for $-1 \leq$ $r \leq 1$ with the equality holding if and only if $x_{1}=\cdots=x_{n}$. This is a generalization of Theorem 1.5.

As a generalization of Theorem 1.2, we have the following corollary.

COROLlARY 3.2. Let $f(x, y)=a x^{p}+b y^{p}-1,0<a \leq b, p \geq 1, x_{i} \in\left[0,(a+b)^{-1 / p}\right]$. For $\alpha \leq 1$, let $F(x)=\ln x$ if $\alpha=0$ or $F(x)=x^{\alpha}$, otherwise. Then for $-1 \leq r \leq p$

$$
0<\Delta_{1, r, \alpha} \leq 1
$$

with equality holding if and only if $x_{1}=\cdots=x_{n}$.

Proof. The first inequality is trivial, and the second inequality for the case $-1 \leq$ $r \leq 1$ follows from (3.3) by noticing $M / m^{\prime} \leq 1$. For $1 \leq r \leq p$, we will prove the case $\alpha=1$ and the general case follows from using the mean value theorem. We define for $1 \leq i \leq n-1$ and $x_{n-i}<x \leq M$

$$
\begin{aligned}
\mathbf{x}_{\mathbf{i}} & =\left(x_{1}, \ldots, x_{n-i}, x, \ldots, x\right), \\
\mathbf{y}_{\mathbf{i}} & =\left(y_{1}, \ldots, y_{n-i}, y, \ldots, y\right), \\
E\left(\mathbf{x}_{i}\right) & =P_{n, r}\left(\mathbf{x}_{i}\right)-A_{n}\left(\mathbf{x}_{i}\right)-P_{n, r}\left(\mathbf{y}_{i}\right)+A_{n}\left(\mathbf{y}_{i}\right),
\end{aligned}
$$

and $E_{i}(x)=E\left(\mathbf{x}_{i}\right)$. 
We need to show that $E_{1}\left(x_{n}\right) \geq 0$, notice first for $x_{1} \leq \cdots \leq x_{n}$

$$
\begin{gathered}
P_{n, r}(\mathbf{x})^{1-r} \cdot x_{n}^{r-1}+P_{n, r}(\mathbf{y})^{1-r} \cdot y_{n}^{r-1} \geq 2\left(\frac{P_{n, r}(\mathbf{x}) P_{n, r}(\mathbf{y})}{x_{n} y_{n}}\right)^{(1-r) / 2}, \\
\frac{P_{n, r}(\mathbf{x}) P_{n, r}(\mathbf{y})}{x_{n} y_{n}}=\left[\sum_{i=1}^{n} \omega_{i}^{2}\left(\frac{x_{i} y_{i}}{x_{n} y_{n}}\right)^{r}+\sum_{1 \leq i<j \leq n} \omega_{i} \omega_{j}\left(\left(\frac{x_{i} y_{j}}{x_{n} y_{n}}\right)^{r}+\left(\frac{x_{j} y_{i}}{x_{n} y_{n}}\right)^{r}\right)\right]^{1 / r} .
\end{gathered}
$$

Since the function $x\left[(1 / b)\left(1-a x^{p}\right)\right]^{1 / p}$ is increasing for $0 \leq x^{p} \leq 1 / 2 a$, we have $\left(x_{i} y_{i} / x_{n} y_{n}\right)^{r} \leq 1$ for all $i$.

Now for fixed $i \leq j$, define

$$
h(x)=2 x_{j}^{r} y_{j}^{r}-y^{r} x_{j}^{r}-x^{r} y_{j}^{r},
$$

then $h^{\prime}\left(x_{i}\right) \leq r x_{i}^{p-1} y_{i}^{r-p} x_{j}^{r}-r x_{i}^{r-1} y_{j}^{r} \leq 0$ since $r \leq p$ and $x_{i} / y_{i} \leq 1 \leq y_{j} / x_{j}$. Thus $h\left(x_{i}\right)=2 x_{j}^{r} y_{j}^{r}-y_{i}^{r} x_{j}^{r}-x_{i}^{r} y_{j}^{r} \geq h\left(x_{j}\right)=0$, which implies

$$
\left(\frac{x_{i} y_{j}}{x_{n} y_{n}}\right)^{r}+\left(\frac{x_{j} y_{i}}{x_{n} y_{n}}\right)^{r} \leq\left(\frac{x_{i} y_{j}}{x_{j} y_{j}}\right)^{r}+\left(\frac{x_{j} y_{i}}{x_{j} y_{j}}\right)^{r}=\left(\frac{x_{i}}{x_{j}}\right)^{r}+\left(\frac{y_{i}}{y_{j}}\right)^{r} \leq 2 .
$$

Back to (3.7), we have

$$
\frac{P_{n, r}(\mathbf{x}) P_{n, r}(\mathbf{y})}{x_{n} y_{n}}=\left(\left(\sum_{i=1}^{n} \omega_{i} \frac{x_{i}^{r}}{x_{n}^{r}}\right)\left(\sum_{i=1}^{n} \omega_{i} \frac{y_{i}^{r}}{y_{n}^{r}}\right)\right)^{1 / r} \leq\left(\sum_{i=1}^{n} \omega_{i}^{2}+\sum_{i<j} 2 \omega_{i} \omega_{j}\right)^{1 / r}=1 .
$$

In particular this gives (where $\Omega_{i}^{-1}=\sum_{k=n-i+1}^{n} \omega_{k}$ )

$$
\begin{aligned}
\Omega_{i}^{-1} E_{i}^{\prime}(x) & =P_{n, r}\left(\mathbf{x}_{i}\right)^{1-r} \cdot x^{r-1}-1+\frac{a}{b} \cdot \frac{x^{p-1}}{y^{p-1}}\left(P_{n, r}\left(\mathbf{y}_{i}\right)^{1-r} \cdot y^{r-1}-1\right) \\
& \geq P_{n, r}\left(\mathbf{x}_{i}\right)^{1-r} \cdot x^{r-1}-1+P_{n, r}\left(\mathbf{y}_{i}\right)^{1-r} \cdot y^{r-1}-1 \geq 0 .
\end{aligned}
$$

Thus we deduce

$$
E_{1}\left(x_{n}\right) \geq E_{1}\left(x_{n-1}\right)=E_{2}\left(x_{n-1}\right) \geq \cdots \geq E_{n-1}\left(x_{2}\right) \geq E_{n-1}\left(x_{1}\right)=0 .
$$

A close look of the proof tells us the equality holds in (3.4) if and only if $x_{1}=$ $\cdots=x_{n}$ and the proof is completed.

As a special case of the above corollary, we have $A_{n}^{\prime}-H_{n}^{\prime} \leq A_{n}-H_{n}$ for generalized weighted means, a proof of this for the special case where $\omega_{1}=\cdots=\omega_{n}$ was given in [2].

We remark here if $0<b<a$, then in general $P_{n, 1}(\mathbf{x})-P_{n, r}(\mathbf{x})$ and $P_{n, 1}(\mathbf{y})-P_{n, r}(\mathbf{y})$ are not comparable. For example, if we let $a=2, b=1, n=2, \omega_{1}=\omega_{2}$, then when $x_{1}=1 / 3, x_{2}=1 / 27, A_{2}-G_{2}=A_{2}^{\prime}-G_{2}^{\prime}$; when $x_{1}=1 / 3, x_{2}=0, A_{2}-G_{2}>A_{2}^{\prime}-G_{2}^{\prime}$ and when $x_{1}=1 / 3, x_{2}=1 / 4, A_{2}-G_{2}<A_{2}^{\prime}-G_{2}^{\prime}$.

The classical case of Ky Fan's inequality corresponds to the choice of $f(x, y)=$ $x+y-1$, where $f_{x}^{\prime} / f_{y}^{\prime}=1$. In this case both inequalities (2.1) and (2.2) hold and combinations of previous results yield the following corollary. 
COROLLARY 3.3. For $f(x, y)=x+y-1,0<m<M \leq 1 / 2, n \geq 2$ then for $-1 \leq r \leq$ $1, \alpha \leq 1$

$$
\left(\frac{m}{1-m}\right)^{2-\alpha}<\Delta_{1, r, \alpha}<\left(\frac{M}{1-M}\right)^{2-\alpha}
$$

4. The comparison of $P_{n, s}^{\alpha}(\mathbf{y})-P_{n, r}^{\alpha}(\mathbf{y})$ and $P_{n, s}^{\alpha}(\mathbf{x})-P_{n, r}^{\alpha}(\mathbf{x})$. In this section, fixing $f(x, y)=x+y-1, x_{i} \in[0,1 / 2]$, we give some results relating the comparison of $P_{n, s}^{\alpha}(\mathbf{y})-P_{n, r}^{\alpha}(\mathbf{y})$ and $P_{n, s}^{\alpha}(\mathbf{x})-P_{n, r}^{\alpha}(\mathbf{x})$, where $s>r, \alpha \in \mathbb{R}$.

Our first result is the following lemma.

LEMMA 4.1. Given $s>r$, if for $\alpha_{0} \in R$, we have $\Delta_{s, r, \alpha_{0}} \leq(\geq) 1$ with equality holding if and only if $x_{1}=\cdots=x_{n}$, then for all $\alpha \leq(\geq) \alpha_{0}, \Delta_{s, r, \alpha} \leq(\geq) 1$ with equality holding if and only if $x_{1}=\cdots=x_{n}$.

Proof. Let $i=s, r, \mathbf{v}=\mathbf{x}, \mathbf{y}$, we can assume $P_{n, i}(\mathbf{v}) \neq 0$. If $\alpha_{0} \neq 0$, write $P_{n, s}^{\alpha}(\mathbf{v})-$ $P_{n, r}^{\alpha}(\mathbf{v})=\left(P_{n, s}^{\alpha_{0}}(\mathbf{v})\right)^{\alpha / \alpha_{0}}-\left(P_{n, r}^{\alpha_{0}}(\mathbf{v})\right)^{\alpha / \alpha_{0}}=\left(\alpha / \alpha_{0}\right) \xi^{\alpha-\alpha_{0}}\left(P_{n, s}^{\alpha_{0}}(\mathbf{v})-P_{n, r}^{\alpha_{0}}(\mathbf{v})\right)$ with $P_{n, r}(\mathbf{v})<$ $\xi<P_{n, s}(\mathbf{v})$, where when $\alpha=0$, we define $\left(P_{n, i}^{\alpha_{0}}(\mathbf{v})\right)^{0 / \alpha_{0}}=\ln P_{n, i}^{\alpha_{0}}(\mathbf{v})$. By taking the quotient, we get the desired result. If $\alpha_{0}=0$, we write $P_{n, i}^{\alpha}(\mathbf{v})=e^{\alpha \ln P_{n, i}(\mathbf{v})}$ and proceed similarly.

For any $s \geq r$, the above lemma enables us to define a number $\sup (\alpha)_{s, r}$ such that $\Delta_{s, r, \alpha} \leq 1$ holds for all $\alpha<\sup (\alpha)_{s, r}$. A special case of this, $\sup (\alpha)_{1,0}=1$ was determined in [5].

The inequality $\Delta_{s, r, \alpha} \geq 1$ seems unusual but indeed it can happen, even for the case $r=1$. Indeed we have the following theorem.

THEOREM 4.2. $\Delta_{s, 1, \alpha} \geq 1$ for $\alpha \geq s \geq 2 ; \Delta_{s, 1, \alpha} \leq 1$ for $1<s \leq 2, \alpha \leq s ; \Delta_{1, r, \alpha} \leq 1$ for $\alpha \leq r<0$, in all cases the equality holds if and only if $x_{1}=\cdots=x_{n}$.

Proof. From Lemma 4.1, it suffices to prove the theorem for $\alpha=s$ or $r$. In this case, for $x \in[0,1 / 2]$, consider the function $f(x)=x^{t}-(1-x)^{t}$ with $f^{\prime \prime}(x)=t(t-$ 1) $\left(x^{t-2}-(1-x)^{t-2}\right)$. By considering the sign $f^{\prime \prime}(x)$ for various $t$ and using corresponding Jensen's inequality: $\sum \omega_{i} f\left(x_{i}\right) \geq(\leq) f\left(\sum \omega_{i} x_{i}\right)$, the above assertions follow.

In Theorem 4.2, by restricting $x_{i} \in[m, M], 0<m<M \leq 1 / 2$, we will get results similar to Corollary 3.3 and we will leave the statements to the reader.

We have omitted the case $0<r<1$ for Theorem 4.2 since we have a stronger result as Corollary 3.2. We point out an interesting phenomena here that when $s=2$, $\Delta_{2,1, \alpha} \geq(\leq) 1$ for $\alpha \geq(\leq) 2$. We also remark here the proof of (1.1) follows by applying Jensen's inequality to the function $\ln x-\ln (1-x)$ for $x \in[0,1 / 2]$.

Notice $P_{n, s}(\mathbf{x})-P_{n, r}(\mathbf{x}) \geq P_{n, s}(\mathbf{y})-P_{n, r}(\mathbf{y})$ does not hold for arbitrary real numbers $s \geq r$, for otherwise we will have $P_{n, s}(\mathbf{x}) / P_{n, r}(\mathbf{x}) \geq P_{n, s}(\mathbf{y}) / P_{n, r}(\mathbf{y})$ which is not true in general according to a nice result by Chen and Wang [8].

THEOREM 4.3. For arbitrary $n, s>r, x_{i} \in(0,1 / 2], \Delta_{s, r, 0} \leq 1$ holds if and only if $|r+s| \leq 3,2^{r} / r \geq 2^{s} / s$ when $r>0, r 2^{r} \leq s 2^{s}$ when $s<0$.

By using Lemma 4.1 and Theorem 4.3, we get the following theorem. 
THEOREM 4.4. $\sup (\alpha)_{s, r} \geq 0$ if $|r+s| \leq 3,2^{r} / r \geq 2^{s} / s$ when $r>0, r 2^{r} \leq s 2^{s}$ when $s<0$. Moreover, $\sup (\alpha)_{1, r}=1$ for $-1 \leq r \leq 1$ and $\sup (\alpha)_{s, 1}=s$ for $1<s \leq 2$.

Proof. The first assertion follows from Theorem 4.3 and the definition for $\sup (\alpha)_{s, r}$. From Corollary 3.2, we know that $\sup (\alpha)_{1, r} \geq 1$ for $-1 \leq r \leq 1$ and when $\alpha>1$, let $x_{1}=1 / 2, x_{2}=\cdots=x_{n}=\epsilon$, and

$$
f\left(\omega_{1}, \epsilon\right)=\left(P_{n, 1}^{\alpha}(\mathbf{x})-P_{n, r}^{\alpha}(\mathbf{x})\right)-\left(P_{n, 1}^{\alpha}(\mathbf{y})-P_{n, r}^{\alpha}(\mathbf{y})\right) .
$$

A simple calculation reveals that there exist positive real numbers $\delta$ and $\eta$ such that we have $f\left(\omega_{1}, \epsilon\right)<0$, if $0<\omega_{1}<\delta$ and $0<\epsilon<\eta$ and $f\left(\omega_{1}, \epsilon\right)>0$, if $1-\delta<$ $\omega_{1}<1$ and $0<\epsilon<\eta$. Similar conclusion holds for $\sup (\alpha)_{s, 1}$ and this completes the proof.

\section{REFERENCES}

[1] H. Alzer, Ungleichungen für geometrische und arithmetische Mittelwerte [Inequalities for geometric and arithmetic means], Nederl. Akad. Wetensch. Indag. Math. 50 (1988), no. 4, 365-374 (German). MR 90a:26034. Zbl 0655.26010.

[2] __ An inequality for arithmetic and harmonic means, Aequationes Math. 46 (1993), no. 3, 257-263. MR 94h:26021. Zbl 0785.26012.

[3] _ The inequality of Ky Fan and related results, Acta Appl. Math. 38 (1995), no. 3, 305-354. MR 96a:26017. Zbl 0834.26013.

[4] _ On an additive analogue of Ky Fan's inequality, Indag. Math. (N.S.) 8 (1997), no. 1, 1-6. MR 99c:26013. Zbl 0873.26009.

[5] _ Some inequalities for arithmetic and geometric means, Proc. Roy. Soc. Edinburgh Sect. A 129 (1999), no. 2, 221-228. MR 2000c:26012. Zbl 0923.26013.

[6] E. F. Beckenbach and R. Bellman, Inequalities, Ergebnisse der Mathematik und ihrer Grenzgebiete, vol. 30, Springer-Verlag, Berlin, 1961. MR 28\#1266. Zbl 0097.26502.

[7] A. McD. Mercer, Bounds for A-G, A-H, G-H, and a family of inequalities of Ky Fan's type, using a general method, J. Math. Anal. Appl. 243 (2000), no. 1, 163-173. MR 2001a:26021. Zbl 0944.26027.

[8] Z. Wang and J. Chen, Generalization of Ky Fan inequality, Math. Balkanica (N.S.) 5 (1991), no. 4, 373-380 (1992). MR 93g:26030. Zbl 0755.26010.

Peng GaO: Department of Mathematics, University of Michigan, 2074 East Hall, 525

EAST UNIVERSITY AVENUE, ANN ARBOR, MI 48109, USA

E-mail address: penggao@umich.edu 


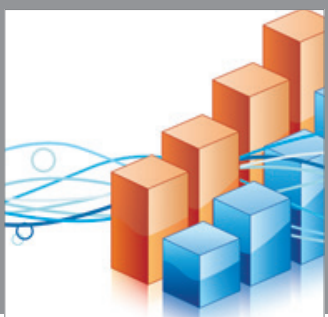

Advances in

Operations Research

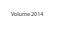

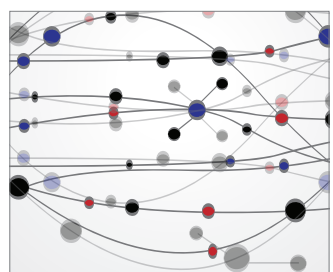

\section{The Scientific} World Journal
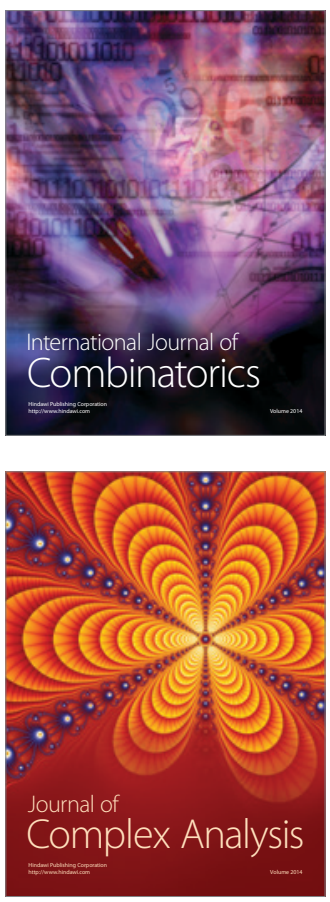

International Journal of

Mathematics and

Mathematical

Sciences
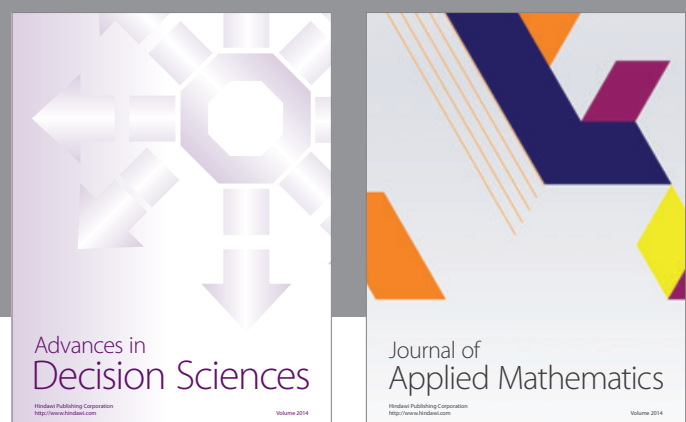

Journal of

Applied Mathematics
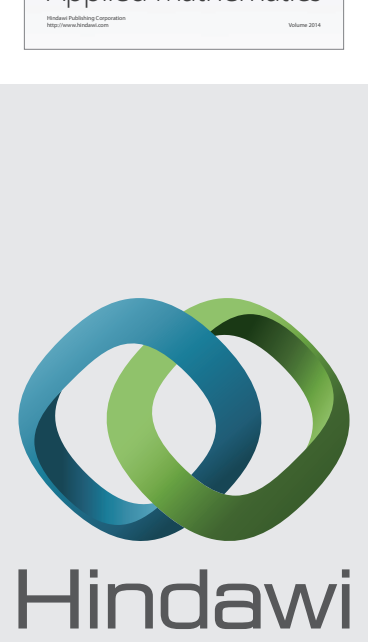

Submit your manuscripts at http://www.hindawi.com
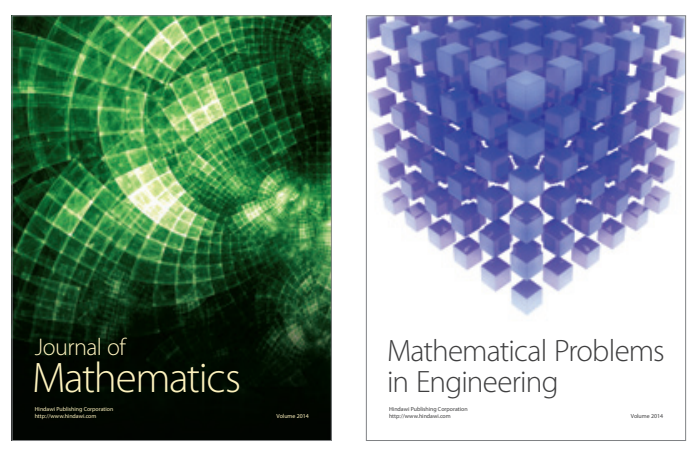

Mathematical Problems in Engineering
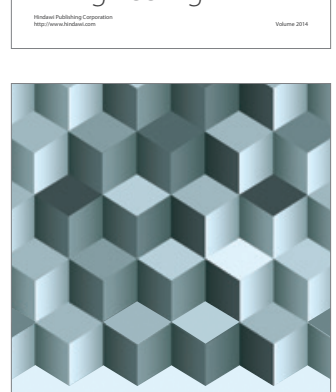

Journal of

Function Spaces
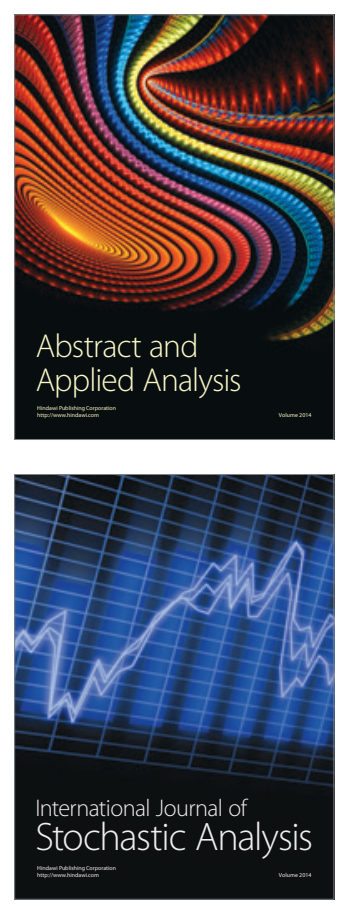

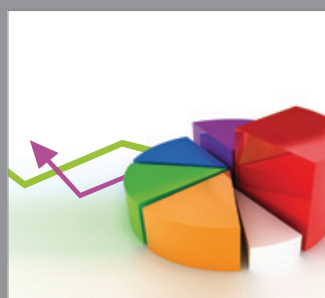

ournal of

Probability and Statistics

Promensencen
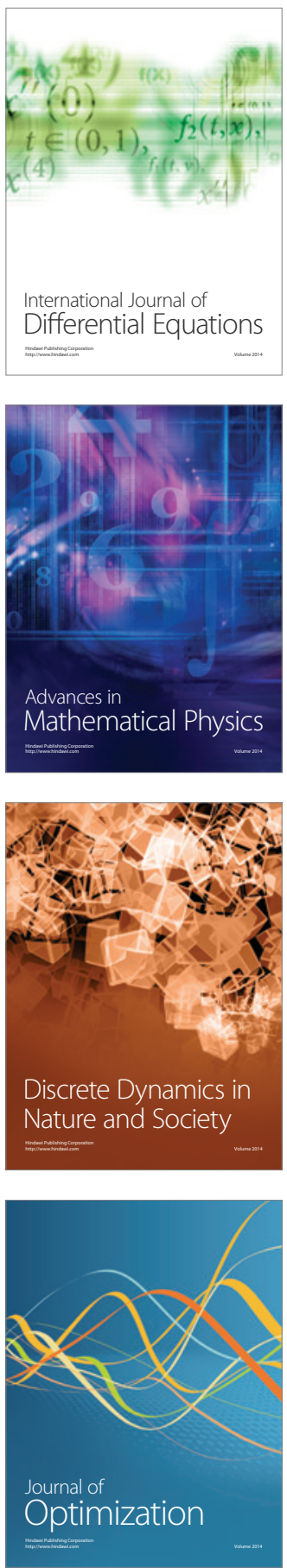